\title{
Ordering kinetics in model systems with inhibited interfacial adsorption
}

\author{
Willart, J.-F.; Mouritsen, Ole G.; Naudts, J.; Descamps, M.
}

Published in:

Physical Review B

Link to article, DOI:

10.1103/PhysRevB.46.8089

Publication date:

1992

Document Version

Publisher's PDF, also known as Version of record

Link back to DTU Orbit

Citation (APA):

Willart, J-F., Mouritsen, O. G., Naudts, J., \& Descamps, M. (1992). Ordering kinetics in model systems with inhibited interfacial adsorption. Physical Review B, 46(13), 8089-8098.

https://doi.org/10.1103/PhysRevB.46.8089

\section{General rights}

Copyright and moral rights for the publications made accessible in the public portal are retained by the authors and/or other copyright owners and it is a condition of accessing publications that users recognise and abide by the legal requirements associated with these rights.

- Users may download and print one copy of any publication from the public portal for the purpose of private study or research.

- You may not further distribute the material or use it for any profit-making activity or commercial gain

- You may freely distribute the URL identifying the publication in the public portal

If you believe that this document breaches copyright please contact us providing details, and we will remove access to the work immediately and investigate your claim 


\title{
Ordering kinetics in model systems with inhibited interfacial adsorption
}

\author{
J.-F. Willart \\ Laboratoire de Dynamique des Cristaux Moléculaires, Université de Lille I, P5, F-59655 Villeneuve d'Ascq, France \\ Ole G. Mouritsen \\ Department of Physical Chemistry, The Technical University of Denmark, Building 206, \\ DK-2800 Lyngby, Denmark \\ J. Naudts \\ Departement Fysica, Universiteit Antwerpen, Universiteitsplein 1, B-2610 Antwerpen, Belgium \\ M. Descamps \\ Laboratoire de Dynamique des Cristaux Moléculaires, Université de Lille I, P5, F-59655 Villeneuve d'Ascq, France
}

(Received 18 February 1992; revised manuscript received 5 June 1992)

\begin{abstract}
The ordering kinetics in two-dimensional Ising-like spin models with inhibited interfacial adsorption are studied by computer-simulation calculations. The inhibited interfacial adsorption is modeled by a particular interfacial adsorption condition on the structure of the domain wall between neighboring domains. This condition can be either hard, as modeled by a singularity in the domain-boundary potential, or soft, as modeled by a version of the Blume-Capel model. The results show that the effect of the steric hindrance, be it hard or soft, is only manifested in the amplitude, $A$, of the algebraic growth law, $R(t) \sim A t^{n}$, whereas the growth exponent, $n$, remains close to the value $n=\frac{1}{2}$ predicted by the classical Lifshitz-Allen-Cahn growth law for systems with nonconserved order parameter. At very low temperatures there is, however, an effective crossover to a much slower algebraic growth. The results are related to experimental work on ordering processes in orientational glasses. It is suggested that the experimental observation of very slow ordering kinetics in, e.g., glassy crystals of cyanoadamantane may be a consequence of low-temperature activated processes which ultimately lead to a freezing in of the structure.
\end{abstract}

\section{INTRODUCTION}

The presence of a foreign component in a system undergoing a nonequilibrium ordering process is known to have a significant influence on the growth law, $R(t) \sim$ $A f(t)$, which describes the temporal evolution of ordering domains of average linear size $R(t)$. The essential time dependence $f(t)$ of the growing domains for a pure system is often found at late stages to be given by an algebraic growth law ${ }^{1}$

$$
R(t) \sim A t^{n},
$$

where the value of the kinetic exponent obeys a remarkable universality and is basically only determined by the conservation laws in effect. A "foreign" component which couples to the order parameter can change the growth law in either a qualitative way by changing the form of $f(t)$ or in a quantitative way by changing the value of the amplitude, $A$. Since most real systems contain impurities, display inhomogeneities, or suffer from imperfections, the ideal asymptotic growth law is often different from the expected algebraic law or is associated with growth exponents which deviate from those predicted by theory or by the principle of universality. Specific situations which have been studied include systems with quenched randomness (the random-field Ising model or systems with quenched dilution) which are known to display logarithmic growth laws, ${ }^{2-5} f(t) \sim(\log t)^{p}$, systems with annealed randomness (dilution) which often effectively display logarithmic growth or algebraic growth with very small exponent values, ${ }^{6-11}$ despite the fact that the annealed randomness should not influence the asymptotic growth behavior. Obviously, it is important to distinguish between foreign components (vacancies, impurities, random fields, second-phase particles) which are mobile and those which are not. ${ }^{9}$ In both cases it is, however, fruitful to consider the foreign component as specifically interacting with the walls between different ordered domains leading to a lowering of the interfacial tension. This is a particular case of dynamic interfacial adsorption. ${ }^{12}$

An interesting situation arises in some pure systems which themselves can create a foreign component during the dynamic ordering process. As an example, consider a system of molecules with internal states of which only one or a few are compatible with the structure of a given ordered domain. The other internal states may, however, be favorable to mediate domain boundaries and are hence created at the boundaries and there act effectively as an interfacially adsorbed species. Among models with this type of behavior are chiral clock models ${ }^{13}$ and axial nextnearest-neighbor Ising models ${ }^{14}$ near the wetting line, the 
Blume-Capel model, ${ }^{15}$ as well as certain Potts models with anisotropic grain-boundary potential. ${ }^{16}$

In the present paper we have conducted a comparative model study of the ordering kinetics in two simple models with intrinsic mechanisms which support interfacial adsorption at the domain boundaries due to self-induction of a foreign component. The work has been inspired by some intriguing experimental results for the ordering kinetics in orientational glasses, such as cyanoadamantane. ${ }^{17-19}$ In this context the self-induced foreign component in the model may be thought of as an orientationally disordered molecule in the glassy crystal. In order to focus on the principles, we have chosen to study the problem within two-dimensional Isinglike lattice models in which the degrees of freedom are not intended to be a realistic description of the details of the orientational molecular variables. It is, however, anticipated that general aspects of the effects of interfacial adsorption on domain growth may be studied via these models. The models have twofold degenerate ordering as in an Ising ferromagnet. We have studied two types of models in which the interfacial adsorption condition (steric hindrance) is enforced either in a hard or a soft manner: (a) Model A with a hard condition: this model is an extension of an earlier model ${ }^{18}$ devised to study the problem. Within this model, domain boundaries have to be wetted by a foreign component which is created subject to a particular applied chemical potential and is supposed to model a disordered molecule. (b) Model B with a soft condition: this model is the wellknown spin-1 Blume-Capel model ${ }^{20}$ in the ferromagnetic part of its phase diagram where spin-0 states are created predominantly at the domain boundaries. We have studied the domain-growth kinetics of both these models using computer-simulation quenching techniques and have compared the results to those of the two-dimensional Ising model with nonconserved order parameter.

In Sec. II we present the models in some detail and describe the simulation techniques we have used. The equilibrium properties of the models are presented in Sec. III with emphasis on the phase transition characteristics. In Sec. IV we describe our results for the nonequilibrium ordering processes in both models and investigate the growth in terms of dynamical structure factors and growth laws. Patterns of the microstructural evolution are presented and evidence in favor of dynamical scaling is put forward. The paper is concluded by a discussion in Sec. V where a comparison is made with the experimental data for the dynamics of orientational ordering in glassy crystals.

\section{MODELS AND SIMULATION TECHNIQUES}

\section{A. Models with hard and soft interfacial adsorption conditions}

The models on which our study are based are extensions of the model proposed by Willart, Descamps, and Naudts ${ }^{18}$ to describe the effects of steric hindrance on domain-growth kinetics. The models are spin-1 Isinglike models arrayed on a square lattice defined by the Hamiltonian

$$
\mathcal{H}=\sum_{\langle i, j\rangle}\left(-J-\frac{K}{2}\left|\sigma_{i}-\sigma_{j}\right|\right) \sigma_{i} \sigma_{j}+D \sum_{i} \sigma_{i}^{2},
$$

where $\sigma_{i}=0, \pm 1$ and the first summation is extended over nearest-neighbor pairs on the square lattice.

The model with the hard condition (model A, steric hindrance) is obtained for $K \rightarrow \infty$ and $J>0$. In this limit, spins with different finite spin values $(\sigma= \pm 1)$ cannot be at nearest-neighbor sites and whenever a domain characterized by $\sigma=1$ meets a domain with $\sigma=-1$, sites with $\sigma=0$ have to wet the boundary completely. The original model by Willart, Descamps, and Naudts ${ }^{18}$ is obtained for $K \rightarrow \infty$ and $J=0$. In the original model the ordering process is driven exclusively by the single-site term ( chemical potential) and there is no energetic advantage of forming compact domains. Hence this model has a transition described by a percolation phenomenon ${ }^{18}$ and the system is all interface.

The model with the soft condition (model B) is obtained for $K=0$ and $J>0$. This is the limit of the Blume-Capel model. ${ }^{20}$ When the ferromagnetic pair interactions are included the model has a stable ferromagnetic phase for $D / J<2$ at low temperatures. During the ordering process subsequent to quenches into the ferromagnetic phase, the pair interaction favors the formation of compact domains. The phase diagram of the BlumeCapel model contains a line of wetting transitions. ${ }^{21}$

\section{B. Simulation techniques}

We have calculated the equilibrium thermodynamic properties of the model with the hard condition for $D / J=0$ and the Blume-Capel model for $D / J=0$ and $D / J=1$ using conventional Monte Carlo simulation techniques. The simulations are performed on finite lattices with $N=L \times L$ sites subject to periodic boundary conditions. Finite-size effects have been assessed by studying different lattice sizes, $L=100,200$, and 300 . The equilibrium properties calculated include the average internal energy per site, $E(T)=N^{-1}\langle\mathcal{H}\rangle$, the occupation variables

$$
q_{\sigma}=N^{-1} \sum_{i} \delta\left(\sigma_{i}-\sigma\right), \quad \sigma=0, \pm 1
$$

the transformed fraction of spins

$$
q=\left\langle q_{+1}+q_{-1}\right\rangle,
$$

and the order parameter

$$
\phi=\left\langle\frac{q_{+1}-q_{-1}}{q}\right\rangle \text {. }
$$

We have also calculated the specific heat

$$
C(T)=\frac{1}{\mathrm{k}_{B} T^{2}}\left(\left\langle\mathcal{H}^{2}\right\rangle-\langle\mathcal{H}\rangle^{2}\right)
$$

and the susceptibility 


$$
\chi(T)=\frac{1}{\mathrm{k}_{B} T}\left(\left\langle\left|\frac{q_{+1}-q_{-1}}{q}\right|^{2}\right\rangle-\left\langle\frac{q_{+1}-q_{-1}}{q}\right\rangle^{2}\right) .
$$

The nonequilibrium ordering dynamics of the models is calculated by Monte Carlo simulation of thermal quenches. The system is prepared in the disordered phase at high temperatures and the temperature is subsequently changed to a value below the phase transition temperature. The dynamics of the ordering process is controlled by the stochastic dynamics on which the particular Monte Carlo algorithm is built. We have used conventional single-site Glauber dynamics, which corresponds to a nonconserved order parameter. The time parameter of the associated Markov process is then interpreted as a physical time which is measured in units of Monte Carlo steps per site (MCS/s).

The temporal evolution of the system is followed both by direct inspection of particular microscopic configurations and by calculation of time-dependent ensemble averages. Since some of the nonequilibrium properties of a system undergoing an ordering process are not selfaveraging, ${ }^{22}$ these ensemble averages have to be performed using results obtained from a large number of independent quenches, typically $20-100$.

We have calculated the excess energy

$$
\Delta E(t)=E(t)-E(T)
$$

where $E(t)$ is the nonequilibrium internal energy and $E(T)$ is the equilibrium internal energy at the temperature to which the system is quenched. $\Delta E(T)$ is a measure of the total nonequilibrium energy bound in the entire domain-boundary network. For localized domain boundaries, this energy is proportional to the total perimeter of the domain-boundary network and hence $\Delta E(t)$ scales as an inverse length scale, $\Delta E(t)^{-1} \sim R(t)$.

A more comprehensive description of the evolving structure is provided by the time-dependent static structure factor, $S(q, t)$, which under the assumption of translational invariance is given by

$$
S(q, t)=\left\langle N^{-1}\left|\sum_{j} \sigma_{j}(t) e^{i q \cdot r_{j}}\right|^{2}\right\rangle .
$$

A quantitative measure of the length scale of the growing ordered domains is obtained via the moments, $k_{m}(t)$, of $S(q, t)$

$$
k_{m}(t)=\sum_{q}{ }^{\prime} q^{m} S(q, t) / \sum_{q}{ }^{\prime} S(q, t),
$$

where the primed sums are restricted by an ultraviolet cutoff. From the moments, measures of the linear length scale may be derived as $R(t) \sim\left(k_{m}\right)^{-d / m}$, where $d=2$ is the spatial dimension. Without an explicit assumption about the shape of the structure factor it is not possible to relate measures of the length scale obtained in this way to lengths in real space.

\section{EQUILIBRIUM PROPERTIES AND PHASE TRANSITIONS}

For both models with $D=0$ the simulation results for the occupation variables, the internal energy, the order parameter, and the transformed fraction as functions of temperature are given in Figs. 1 and 2. The data for the specific heat and the compressibility are displayed in Figs. 3 and 4 . The data refer to a $100 \times 100$ lattice. Since the main issue of the present paper is to study the nonequilibrium ordering processes, we have not performed a full-fledged finite-size scaling analysis of the transition properties of either of the two models. The transition in the two models is most directly identified by the pronounced peak in the isothermal susceptibility in Fig. 4. The transition temperatures found for the models are in good agreement with previous estimates. ${ }^{21,23}$ It is seen from the data in Fig. 2 that the hard condition leads to a stabilization of the ordered ferromagnetic phase and the transition temperature of model A is more than twice as high as that of model B. Furthermore, the transition region is more narrow in model $\mathrm{B}$. The transition is barely reflected in the variation of the internal energy in the model with the hard condition. The specific heat gives hardly any sign of the transition in model A
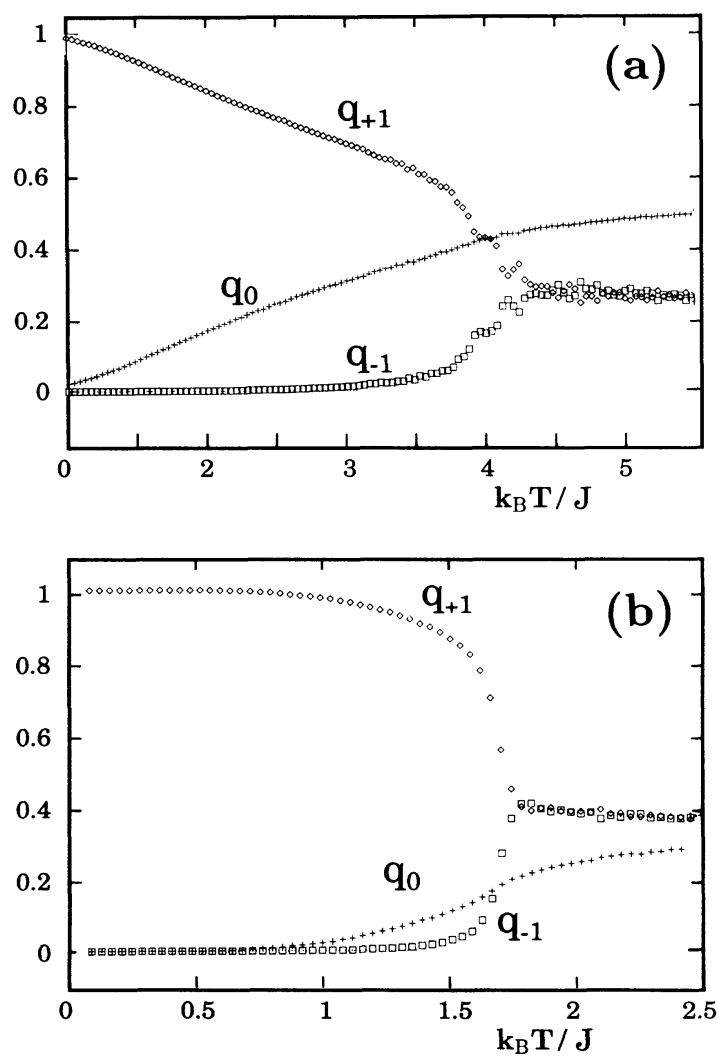

FIG. 1. Equilibrium results for the occupation variables, $q_{\sigma}(T)$ in Eq. (3), for the model with the hard condition (a) and the model with the soft condition (b) in the case $D=0$, Eq. (2). $\diamond: q_{+1}(T), \square: q_{-1}(T)$, and $+: q_{0}(T)$. The data refer to a simulation on a lattice with $100 \times 100$ sites. 

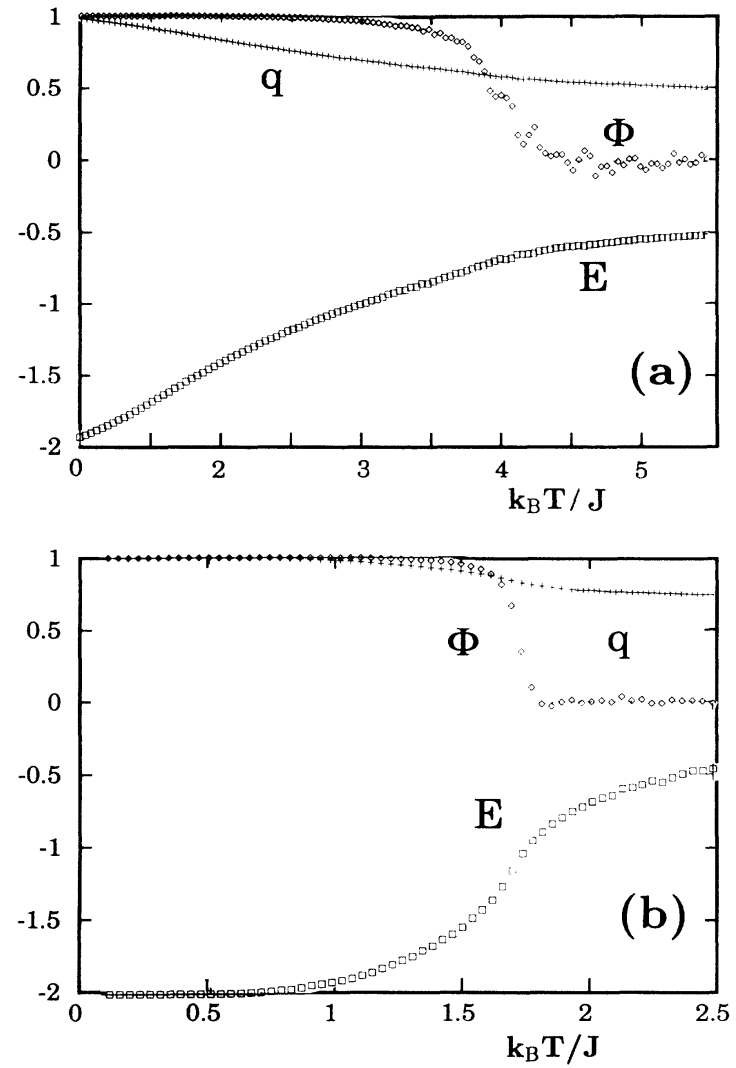

FIG. 2. Equilibrium results for order parameter, $\phi(T)$ in Eq. (5), the average internal energy, $E(T)$, and the transformed fraction, $q(T)$ in Eq. (4), for the model with the hard condition (a) and the model with the soft condition (b) in the case $D=0$, Eq. (2). $\diamond: \phi(T), \square: E(T)$, and $+: q(T)$. The data refer to simulations on a lattice with $100 \times 100$ sites.

whereas the specific heat has a dramatic peak at the transition in model B, cf. Fig. 3. Hence the transition in model $\mathrm{B}$ is driven purely by entropy. The much smoother energy variation in model $B$ is due to the fact that excitations in the model with the hard condition are much more costly since, in order to reverse a spin in the ferromagnetic phase, a cluster of at least four sites with $\sigma=0$ has to be created before a spin fully wetted by $\sigma=0$ states can flip. This is clearly borne out by the temperature variation of the occupation variables in Fig. 1, which shows that $q_{0}$ rises to a very high value in the ordered phase as the transition is approached, whereas $q_{0}$ in the Blume-Capel model only gains some weight in the transition region. It should be noted that the asymmetry in Fig. $1, q_{+1} \neq q_{-1}$, below the transition is due to the symmetry breaking in the ordered phase, which in this case corresponds to a ferromagnetic state in the $\sigma=1$ manifold.

\section{NONEQUILIBRIUM ORDERING AND GROWTH}

\section{A. Microscopic evolution}

In Fig. 5 is shown a selection of snapshots of microconfigurations typical of thermal quenches of the two models from high temperatures to low temperatures within the ferromagnetic phase. Since this phase is twofold degenerate, two types of domains are spontaneously nucleated as the system responds to have been brought into the thermodynamically unstable regime. The hard condition of model $\mathrm{A}$ implies that the domain walls are always wetted by spins in the $\sigma=0$ state. In model $\mathrm{B}$, which corresponds to a soft condition for the interfacial adsorption, the two types of ferromagnetically ordered domains can meet but it is obvious from the snapshots that the system has a distinct tendency to wet the interfaces by $\sigma=0$ spins. The overall conclusion obtained from comparing the two panels of snapshots in Fig. 5 is that the domain-growth kinetics is much slower in model $\mathrm{A}$ than in model B. The adsorption at the domain-boundary network lowers the interfacial tension and slows down the driving force for the interface motion. However, as we shall show below, only the absolute growth rate is slowed down. The essential time-dependent growth function, $f(t)$, is in fact the same in the two models.
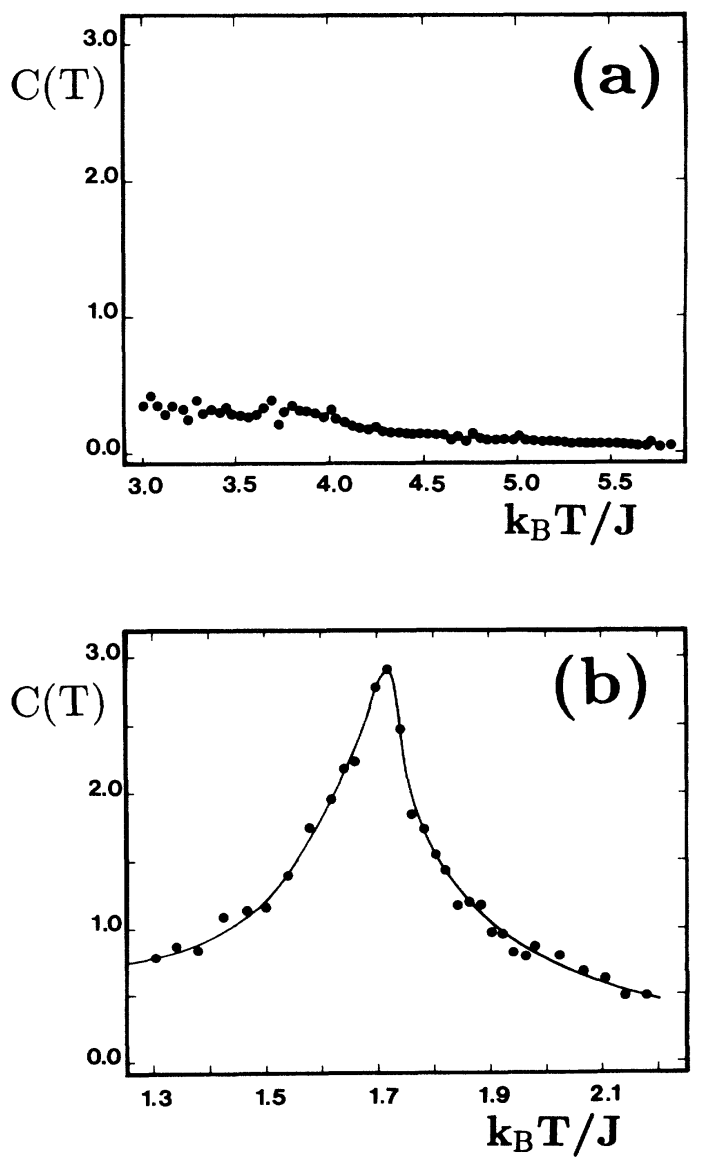

FIG. 3. Equilibrium results for specific heat, $C(T)$ in Eq. (6), for the model with the hard condition (a) and the model with the soft condition (b) in the case $D=0$, Eq. (2). The data refer to simulations on a lattice with $100 \times 100$ sites. 

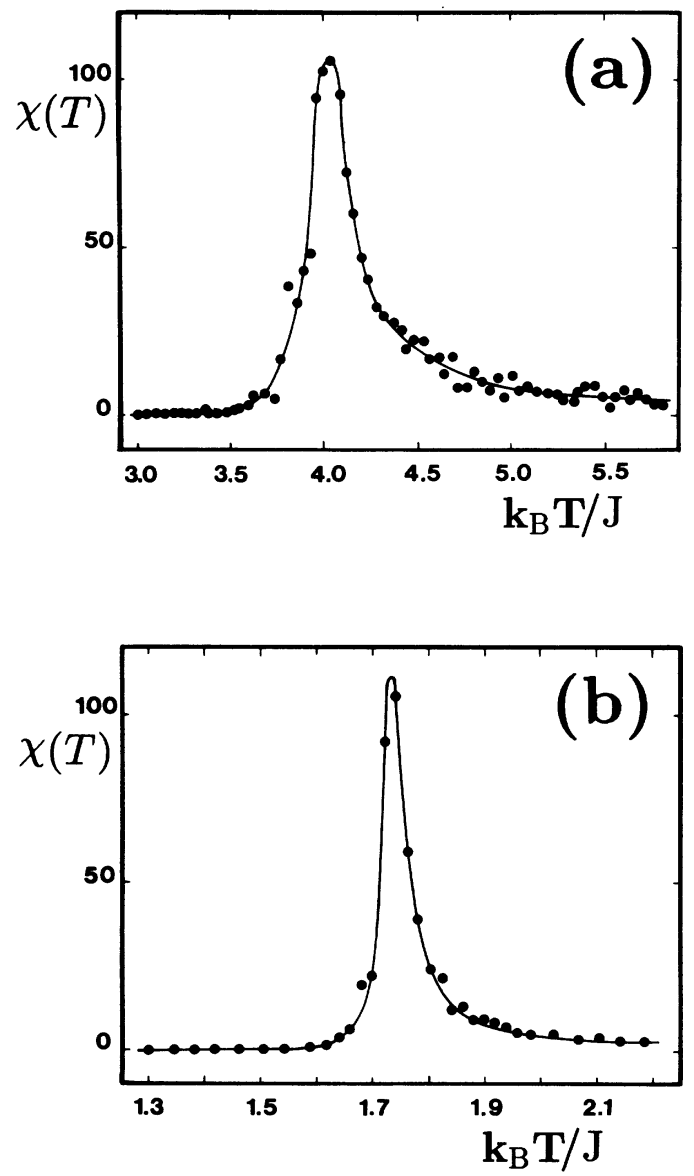

FIG. 4. Equilibrium results for isothermal susceptibility, $\chi(T)$ in Eq. (7), for the model with the hard condition (a) and the model with the soft condition (b) in the case $D=$ 0 , Eq. (2). The data refer to simulations on a lattice with $100 \times 100$ sites.

\section{B. Structure factor and dynamical scaling}

The simulation data for the time-dependent static structure factor, $S(q, t)$ in Eq. (9), for the two models with $D=0$ are displayed in Fig. 6. Comparison of the structure factor for the two models shows that the growth is much slower in model A than in model B. A time-dependent length scale may be extracted from the time-dependent static structure factor via the moments, Eq. (10). We shall return to the moments in Sec. IV C below.

We have tested whether the ordering processes in the two models obey dynamical scaling by constructing the dynamical scaling function, $F(x)$, and the scaling variable, $x$, as

$$
F(x)=S(q, t) / k_{1}^{2}(t), \quad x=q k_{1}^{-1} .
$$

The dynamical scaling functions for the two models are shown in Fig. 7. In both cases it is seen that the ordering process within statistical accuracy obeys dynamical scaling in an extended temperature regime. By analysis of the tails of the scaling functions we have confirmed that the Porod law,

$$
F(x) \sim x^{-(d+\omega)}, \quad q \gg 1
$$

holds with $\omega \simeq 1$ as expected for small-angle scattering on domains with sharp boundaries. ${ }^{24}$ We have also constructed other scaling functions, e.g., $S(q, t) / S(q=0, t)$ and $S(q, t) / k_{2}(t)$, and found that the data are equally consistent with the existence of such functions.

\section{Growth laws}

The time dependence of the different measures of inverse length scale, $\Delta E(t), k_{1}(t)$, and $k_{2}^{1 / 2}$, is analyzed quantitatively for the two models in Figs. 8-10. Data for different quench temperatures are shown. The overall picture which emerges from these sets of data is as follows.

In model $\mathrm{A}$ the growth stops after a very short time at $T=0$ and the domain pattern freezes in. As the temperature is raised, the system grows with an earlytime effectively algebraic growth law with an exponent which increases with increasing temperature. At intermediate times there is a crossover to a late-time growth (a)

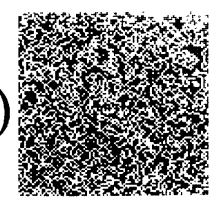

$t=5$

(b)

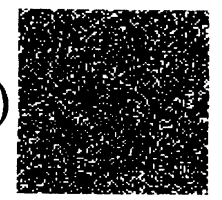

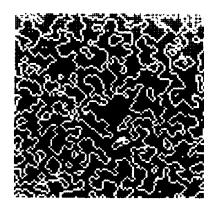

$\mathbf{t}=\mathbf{2 0}$

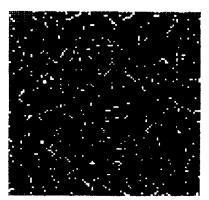

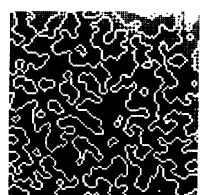

$\mathbf{t}=\mathbf{1 0 0}$

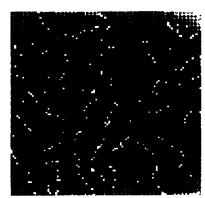

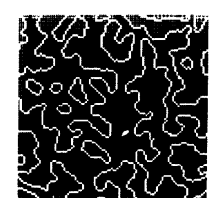
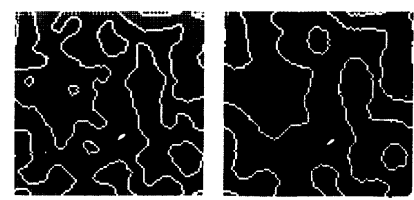

$$
\mathbf{t}=\mathbf{5 0 0}
$$

$\mathbf{t}=\mathbf{2 0 0 0}$
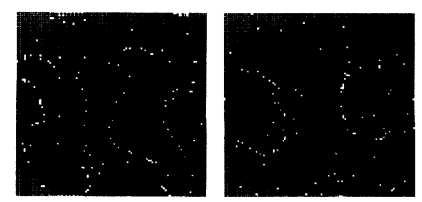

$\mathbf{t}=\mathbf{5 0 0 0}$

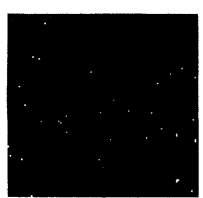

FIG. 5. Snapshots of typical microconfigurations illustrating the temporal evolution of the ordering subsequent to a deep thermal quench from $\mathrm{k}_{B} T / J=\infty$. (a) corresponds to the model with the hard condition quenched to $\mathrm{k}_{B} T / J=0.5$ and (b) to the model with the soft condition quenched to $\mathrm{k}_{B} T / J=0.1$. For both models $D=0$ in Eq. (2). The data refer to simulations on a lattice with $100 \times 100$ sites. Sites with $\sigma=0$ are indicated by blanks and sites with $\sigma= \pm 1$ are indicated in two grey tones. The time, $t$, is given in units of $\mathrm{MCS} / \mathrm{s}$. 
behavior also characterized by an algebraic growth law with an exponent which is approximately independent on temperature. The higher the temperature, the earlier is the occurrence of this crossover. The late-time exponent, $n$ in Eq. (1), is different for the three different quantities. For model $\mathrm{A}$ we find that $n \simeq 0.4-0.5$ in the case of $R(t) \sim \Delta E^{-1}(t), n \simeq 0.35-0.5$ in the case of $R(t) \sim k_{1}^{-1}(t)$, and $n \simeq 0.25-0.3$ in the case of $R(t) \sim k_{2}^{-1 / 2}(t)$. We have also investigated the growth behavior of other system properties. For example, the variation of the transformed fraction, $\Delta q(t)=q(t)-q(T)$, is found to scale the same way as $\Delta E(t)$.

In model $\mathrm{B}$ the growth persists at all temperatures, even at $T=0$. This is in contrast to the freezing-in behavior of model A at low temperatures. Moreover the growth in model B is well described by a single algebraic growth law for all temperatures and the same growth exponent applies independent of temperature. Similar to
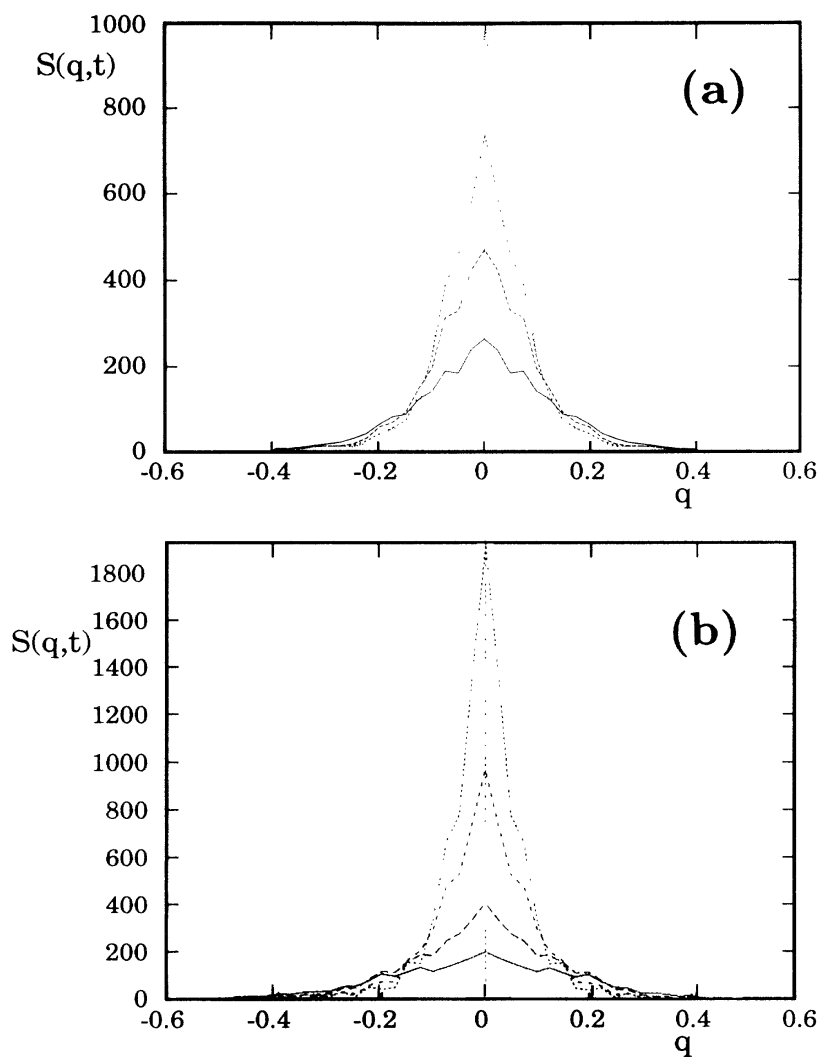

FIG. 6. Time-dependent static structure factor, $S(q, t)$ in Eq. (9), for the model with the hard condition (a) and the model with the soft condition (b) in the case $D=0$, Eq. (2). (a) The model is quenched from $\mathrm{k}_{B} T / J=\infty$ to 2. Data at different times (in units of MCS/s) are indicated by (-): $t=500,(---): t=1000,(---)$ : $t=1500$, and (- - - - ): $t=2000$. The data refer to simulations on a lattice with $200 \times 200$ sites. (b) The model is quenched from $\mathrm{k}_{B} T / J=\infty$ to 0.7 . Data at different times (in units of $\mathrm{MCS} / \mathrm{s}$ ) are indicated by (-): $t=100,(--\longrightarrow): t=200$, $(---): t=500$, and (- - - $): t=1000$. The data refer to simulations on a lattice with $100 \times 100$ sites.
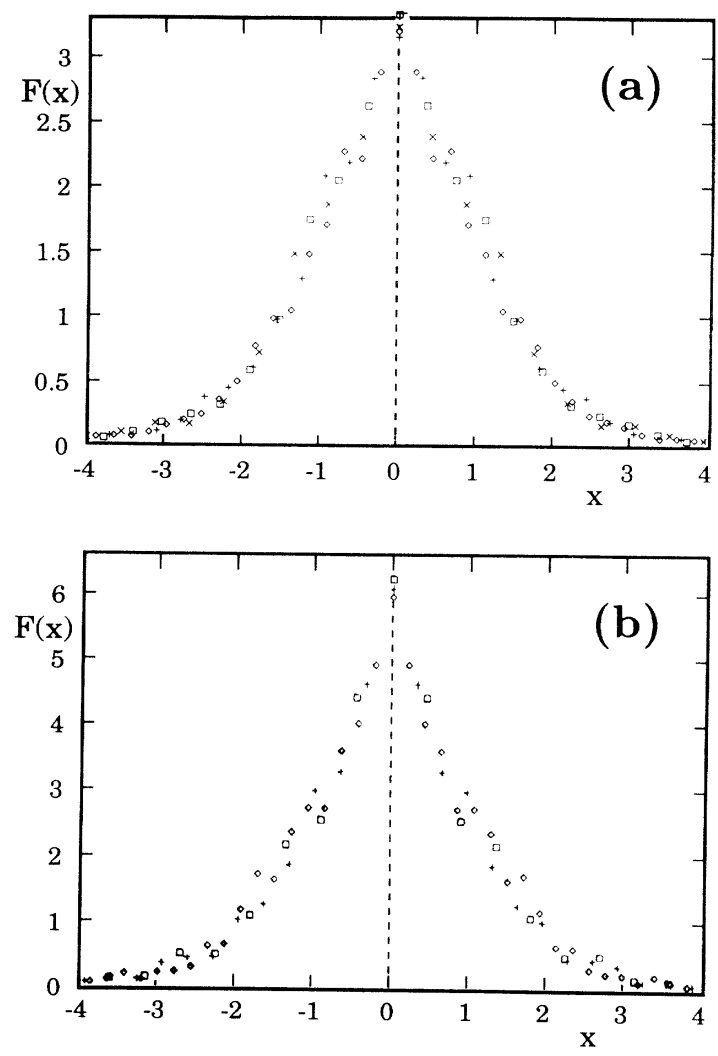

FIG. 7. Dynamical scaling functions, $F(x)$ in Eq. (11), corresponding to the data in Fig. 6. (a) Data at different times (in units of MCS/s) are indicated by $(\diamond): t=500,(+)$ : $t=1000,(\square): t=1500$, and $(\times): t=2000$. (b) Data at different times (in units of MCS/s) are indicated by $(\diamond)$ : $t=200,(+): t=500$, and ( $\square): t=1000$.

model $A$ the value of the growth exponent depends on the quantity under consideration. For model $\mathrm{B}$ we find that $n \simeq 0.4-0.5$ in the case of $R(t) \sim \Delta E^{-1}(t), n \simeq 0.4$ in the case of $R(t) \sim k_{1}^{-1}(t)$, and $n \simeq 0.3-0.35$ in the case of $R(t) \sim k_{2}^{-1 / 2}(t)$. These exponent values are within the numerical accuracy the same as those found for the late-stage growth in model $\mathrm{A}$. We have also studied the growth kinetics in model $\mathrm{B}$ in the case of $D / J=1$ in Eq. (2) and found that the same growth behavior prevails with the same exponent values.

For both models A and B we find that as the quench temperature is increased towards the equilibrium transition temperature there is a new crossover (not shown in the figures) to an effectively algebraic growth law with a smaller exponent. This behavior is caused by critical slowing down which involves ${ }^{25}$ the dynamic critical exponent $z$.

In order to further investigate the significance of our finding of algebraic growth laws for both model A and model $B$ with exponent values which depend on the quantity under consideration, we have investigated the domain-growth kinetics in the ferromagnetic twodimensional Ising model which is obtained from Eq. (2) for $K=D=0$. The ordering kinetics of this model 
has been studied by a number of techniques ${ }^{26}$ and is well understood in terms of the classical theories of curvaturedriven growth of continuous ordering processes with nonconserved order parameter. Specifically, the ordering process is obeying dynamical scaling and the growth law is algebraic with an exponent value $n=\frac{1}{2}$. In Fig. 11 are shown our simulation results for the Ising model for the same three different measures of inverse length scale as calculated for models A and B in Figs. 8-10. A remarkable similarity is observed between the growth laws for all three models. For the Ising model we find, in the temperature and time regimes investigated, that
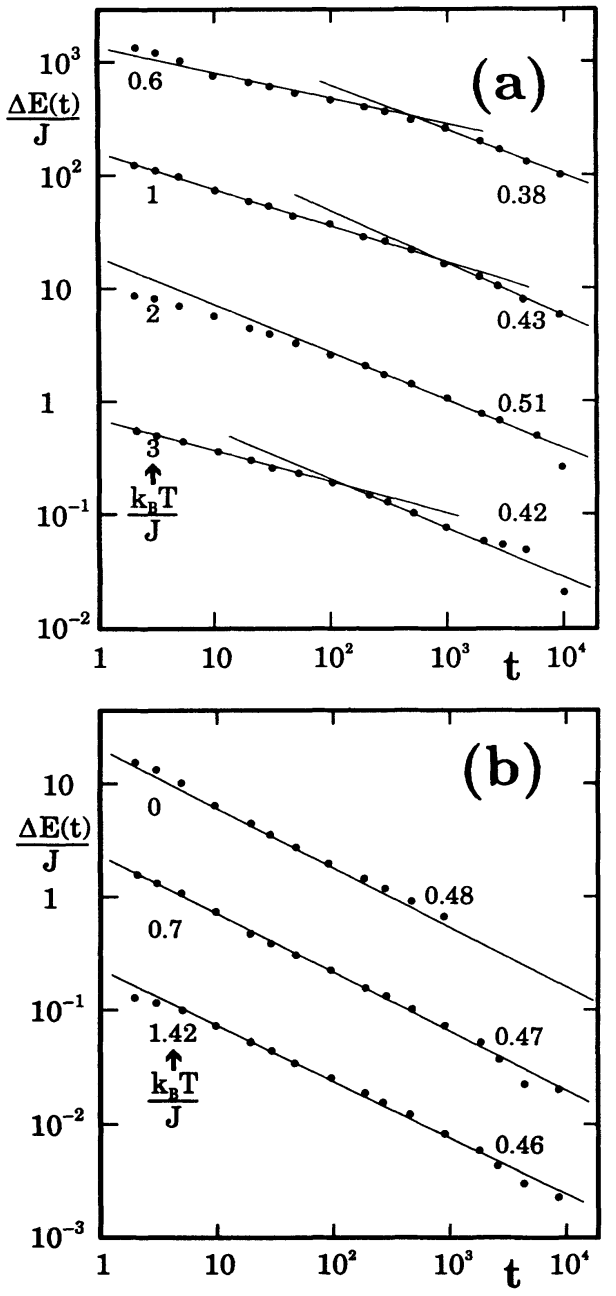

FIG. 8. Log-log plot of the excess internal energy, $\Delta E(t)$ in Eq. (8), vs time, $t$ in units of MCS/s, for the model with the hard condition (a) and the model with the soft condition (b) in the case $D=0$, Eq. (2). The quench temperatures are indicated to the left in the figure. The bottom set of data corresponds to the units on the left-hand axis whereas each of the other data sets is translated one decade above the one below. The solid lines are guides to the eye and the numbers indicated in association with these lines denote their slope. The data refer to simulations on a lattice with $200 \times 200$ sites for model $\mathrm{A}$ and $100 \times 100$ sites for model B. $n \simeq 0.5$ in the case of $R(t) \sim \Delta E^{-1}(t), n \simeq 0.4$ in the case of $R(t) \sim k_{1}^{-1}(t)$, and $n \simeq 0.35$ in the case of $R(t) \sim k_{2}^{-1 / 2}(t)$.

\section{CONCLUSIONS AND DISCUSSION}

We have conducted a comparative study of the ordering kinetics in two different two-dimensional models undergoing a ferromagnetic ordering process in response to thermal quenches below a phase transition. The two
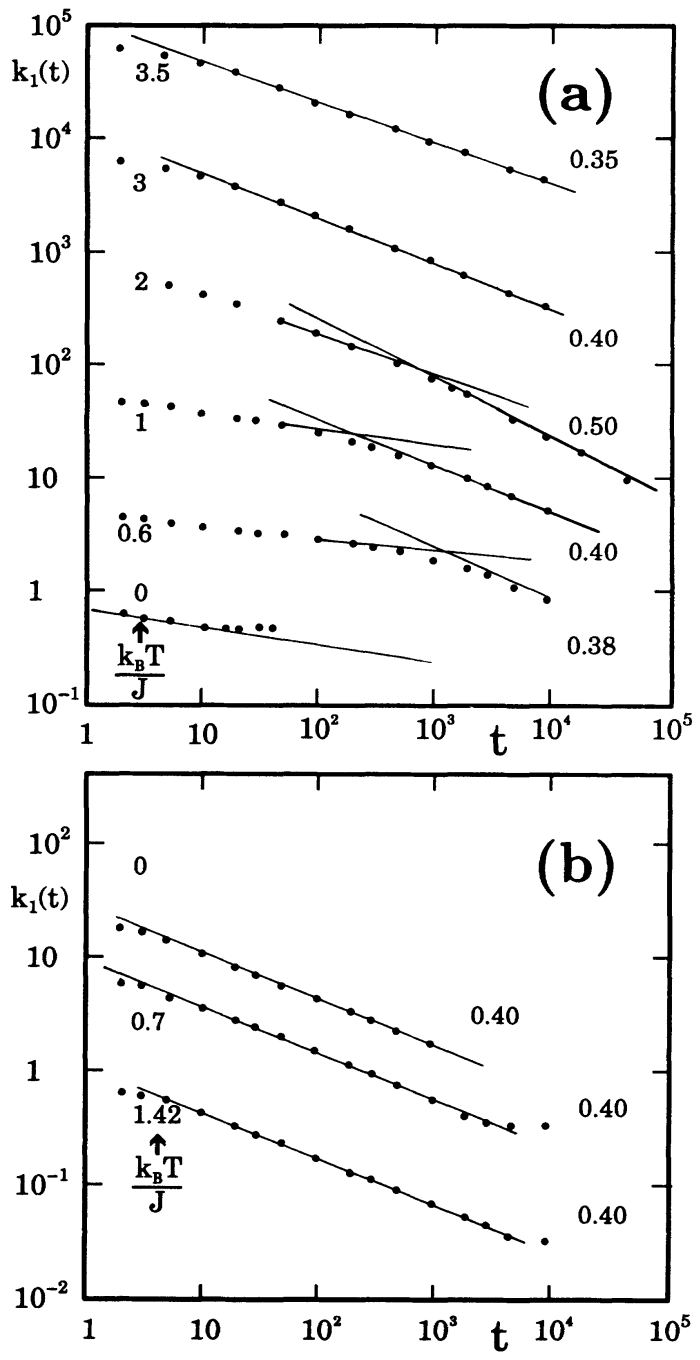

FIG. 9. Log-log plot of the first moment, $k_{1}(t)$ in Eq. (10), of the time-dependent static structure factor vs time, $t$ in units of MCS/s, for the model with the hard condition (a) and the model with the soft condition (b) in the case $D=0$, Eq. (2). The quench temperatures are indicated to the left in the figure. The bottom set of data corresponds to the units on the left-hand axis whereas each of the other data sets is translated one decade above the one below. The solid lines are guides to the eye and the numbers indicated in association with these lines denote their slope. The data refer to simulations on a lattice with $200 \times 200$ sites for model $A$ and $100 \times 100$ sites for model B. 
models, model A and model B, are distinguished by a particular property of the domain walls. The domain walls of model A are formed subject to a hard condition for the interfacial adsorption of a spin- 0 state. In this model the interfaces are completely wetted by particles in the spin-0 state and the domain-boundary dynamics has to adapt to this hard condition. In model B, which is equivalent to the Blume-Capel model, spin-0 states are adsorbed at the domain boundaries but the condition for adsorption is soft in the sense that the interfacial adsorption is controlled by the interfacial tension in the usual way.

Our main result for the ordering dynamics is that the
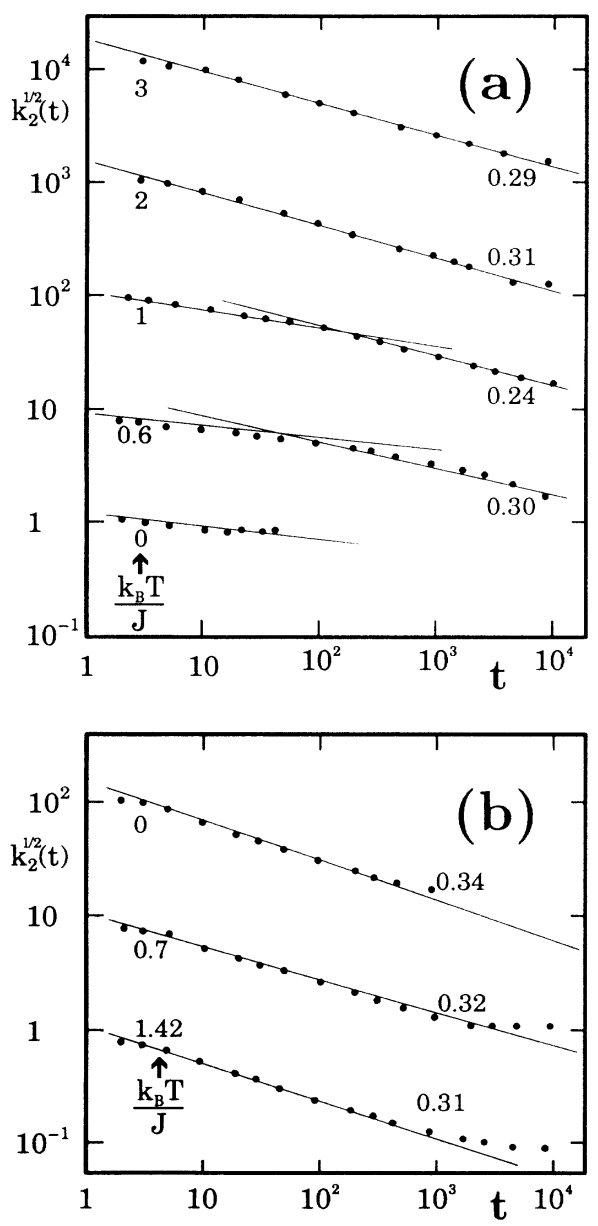

FIG. 10. Log-log plot of the square root of the second moment, $k_{2}^{1 / 2}(t)$ in Eq. (10), of the time-dependent static structure factor vs time, $t$ in units of MCS/s, for the model with the hard condition (a) and the model with the soft condition (b) in the case $D=0$, Eq. (2). The quench temperatures are indicated to the left in the figure. The bottom set of data corresponds to the units on the left-hand axis whereas each of the other data sets is translated one decade above the one below. The solid lines are guides to the eye and the numbers indicated in association with these lines denote their slope. The data refer to simulations on a lattice with $200 \times 200$ sites for model A and $100 \times 100$ sites for model B.

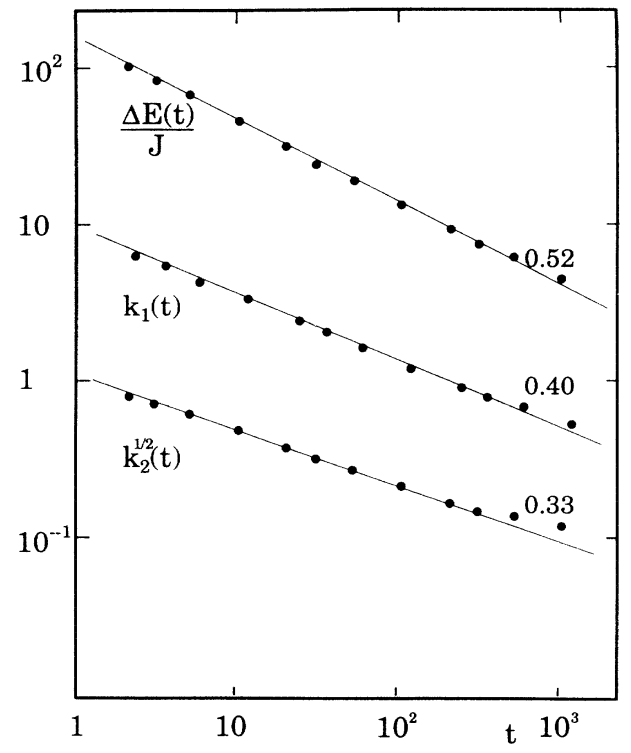

FIG. 11. Log-log plot of the excess energy, $\Delta E(t)$ in Eq. (8), the first moment, $k_{1}(t)$ in Eq. (10), and the square root of the second moment, $k_{2}^{1 / 2}(t)$ in Eq. (10), of the timedependent static structure factor vs time, $t$ in units of MCS/s, for the two-dimensional ferromagnetic Ising model. The bottom set of data corresponds to the units on the left-hand axis whereas each of the other data sets is translated one decade above the one below. The model is quenched from $\mathrm{k}_{B} T / J=\infty$ to 0.9 . The solid lines are guides to the eye and the numbers indicated in association with these lines denote their slope. The data refer to simulations on a lattice with $100 \times 100$ sites.

absolute growth rate in model $\mathrm{A}$ is much slower than in model $\mathrm{B}$. The absolute growth rate is controlled by the prefactor, $A(T)$, in the growth law. The growth processes in both models obey dynamical scaling. Whereas the absolute growth rates in the two models are rather different, the essential time dependence of the growth law, $f(t)$, is the same for both models, $f(t) \sim t^{n}$, with a growth exponent value which in the case of the excess energy is $n \simeq 0.4-0.5$. This value of the growth exponent is consistent with the classical value for Lifshitz-AllenCahn growth kinetics, $n=\frac{1}{2}$, for continuous ordering with nonconserved order parameter. ${ }^{1}$ We have verified that this is also the exponent value which, in accordance with expectations, applies to the domain-growth kinetics of the conventional two-dimensional Ising ferromagnet with nonconserved order parameter (Glauber dynamics).

A striking result of our studies of the ordering kinetics in models $\mathrm{A}$ and $\mathrm{B}$ is that the exponent values which one obtains when analyzing the time dependence of length scales derived from the moments of the time-dependent static structure factor are somewhat lower that the classical value, $n=\frac{1}{2}$, obtained from the excess energy. This seems to indicate a breakdown of dynamical scaling, which implies that different measures of length scale should scale the same way, i.e., $\Delta E^{-1}(t) \sim k_{1}^{-1}(t) \sim$ $k_{2}^{-1 / 2}(t) \sim t^{n}$. However, dynamical scaling seems ef- 
fectively to hold, judging from the analysis of the timedependent static structure factor in Fig. 7. We therefore interpret our finding of systematic lower exponent values from the moments, as well as the finding that the exponent value describing $k_{1}^{-1}(t)$ is consistently larger than that describing $k_{2}^{-1 / 2}(t)$, as indicating that the moments are not proper measures of the length scale. This conclusion implies that a careful analysis of the shape of the time-dependent static structure factor has to be performed in order to identify a relation which permits determination of the coherence length from the structure factor. Our numerical data are not sufficiently accurate to permit such a detailed analysis of the shape of $S(q, t)$. As additional evidence in favor of considering the moments as inappropriate measures of length scale, we wish to point to our results for the time dependence of the moments of the structure factor for the Ising model in Fig. 11. This figure shows quite clearly that even for this well-studied model the growth exponents describing the moments are distinctly lower than that describing the excess energy. In fact, the values of the exponents for $k_{1}^{-1}(t)$ and $k_{2}^{-1 / 2}(t)$ are the same as those found for models $\mathrm{A}$ and $\mathrm{B}$. It is noteworthy that only a few data are available in the literature on the scaling properties of the moments of the time-dependent static structure factor of the Ising model, and the existing data ${ }^{27}$ are rather imprecise and not inconsistent with the results of the present work. Finally, we wish to point out the possibility that moments in general may be inappropriate measures of length scale in ordering processes to the extent that the shape of the structure factor has not been determined. Studies of ordering kinetics in several other models ${ }^{24}$ have also revealed that in those cases the exponent values pertinent for the moments tend to be smaller than those describing length scales derived from, for example, the excess energy or the domain-size distribution function.
The present model study was motivated by the experimental finding of extremely slow domain-growth kinetics in orientational glasses such as cyanoadamantane and related mixed compounds. ${ }^{17-19}$ In these compounds the molecules are positioned in a perfect crystalline array and the orientational ordering process among the bulky, anisotropic molecules is hampered by steric hindrance. The orientational ordering in cyanoadamantane is sixfold degenerate. It has been suggested ${ }^{18}$ that the steric hindrance may be seen as a particular boundary condition for the domain walls separating domains of different orientational order. This condition should imply that the walls are mediated by disordered molecules, and domains of different orientational order cannot meet without induction of an interfacial layer of disordered molecules. In the present model study we have incorporated this condition as a hard condition for an interfacial adsorption process in the simplest possible setting. Our finding of low absolute growth rates, but otherwise classical growth laws, suggests that the experimentally observed algebraic growth laws with anomalously small exponent values may be a consequence of a low-temperature condition. However, the experimental situation is still puzzling. At low temperatures the hard condition, as demonstrated by our comparative model study, implies that the domain-wall migration is an activated process which at low temperatures can lead to arbitrarily small exponents whose values are strongly temperature dependent. At very low temperatures the ordering process leads to a freezing in of the domain pattern at very early times.

\section{ACKNOWLEDGMENTS}

This work was supported by EEC Science contract No. SC1-0426-C(CD) and by the Danish Natural Science Research Council under Grant No. J.nr. 11-7785.
${ }^{1}$ O. G. Mouritsen, in Kinetics of Ordering and Growth at Surfaces, edited by M. G. Lagally (Plenum, New York, 1990), p. 1.

${ }^{2}$ G. Grinstein and J. F. Fernandez, Phys. Rev. B 29, 6389 (1984).

${ }^{3}$ D. A. Huse and C. L. Henley, Phys. Rev. Lett. 54, 2708 (1985).

${ }^{4}$ G. S. Grest and D. J. Srolovitz, Phys. Rev. B 32, 3014 (1985).

${ }^{5}$ D. P. Belanger, A. R. King, and V. Jaccarino, Phys. Rev. B 31, 4538 (1985); H. Yoshizawa, R. A. Cowley, G. Shirane, and R. Birgeneau, ibid. 31, 4548 (1985).

${ }^{6}$ D. J. Srolovitz and G. N. Hassold, Phys. Rev. B 35, 6901 (1987).

${ }^{7}$ R. F. Shannon, C. R. Harkless, and S. E. Nagler, Phys. Rev. B 38, 9327 (1988).

${ }^{8}$ J.-K. Zuo, G.-C. Wang, and T.-M. Lu, Phys. Rev. Lett. 60, 1053 (1988).

${ }^{9}$ O. G. Mouritsen and P. J. Shah, in Kinetics of Ordering and Growth at Surfaces (Ref. 1), p. 45.
${ }^{10}$ P. J. Shah and O. G. Mouritsen, Phys. Rev. B 41, 7003 (1990).

${ }^{11}$ K. Yaldram and K. Binder, Z. Phys. B 82, 405 (1991).

${ }^{12} \mathrm{~W}$. Selke, in Static Critical Phenomena in Inhomogeneous Systems, edited by A. Pejkalski and J. Sznaid (SpringerVerlag, New York, 1984), p. 191.

${ }^{13}$ J. Houlrik and S. J. K. Jensen, Phys. Rev. B 34, 7828 (1986).

${ }^{14}$ T. Ala-Nissila, J. D. Gunton, and K. Kaski, Phys. Rev. B 37, 179 (1988).

${ }^{15}$ H. Röder, D.Phil. Thesis, University of Oxford, 1988 (unpublished).

${ }^{16}$ C. Jeppesen, H. Flyvbjerg, and O. G. Mouritsen, Phys. Rev. B 40, 9070 (1989).

${ }^{17}$ M. Descamps and C. Caucheteux, J. Phys. C 20, 5073 (1987).

${ }^{18}$ J.-F. Willart, M. Descamps, and J. Naudts, Phase Trans. 31, 261 (1991).

${ }^{19}$ M. Descamps and J.-F. Willart, Phase Trans. 31, 247 (1991). 
${ }^{20}$ M. Blume, Phys. Rev. 141, 517 (1966); H. W. Capel, Physica 32, 966 (1966).

${ }^{21}$ H. H. Lee and D. P. Landau, Phys. Rev. B 20, 2893 (1979).

${ }^{22}$ A. Milchev, K. Binder, and D. W. Heermann, Z. Phys. B 61, 521 (1986).

${ }^{23}$ Y.-L. Wang, F. Lee, and J. D. Kimel, Phys. Rev. B 36, 8945 (1987).
${ }^{24}$ O. G. Mouritsen and E. L. Praestgaard, Phys. Rev. B 38, 2703 (1988).

${ }^{25}$ A. Sadiq and K. Binder, J. Stat. Phys. 35, 517 (1984).

${ }^{26}$ For a list of references, see Ref. 1.

${ }^{27}$ P. S. Sahni, G. Dee, J. D. Gunton, M. Phani, J. L. Lebowitz, and M. Kalos, Phys. Rev. B 24, 410 (1981). 


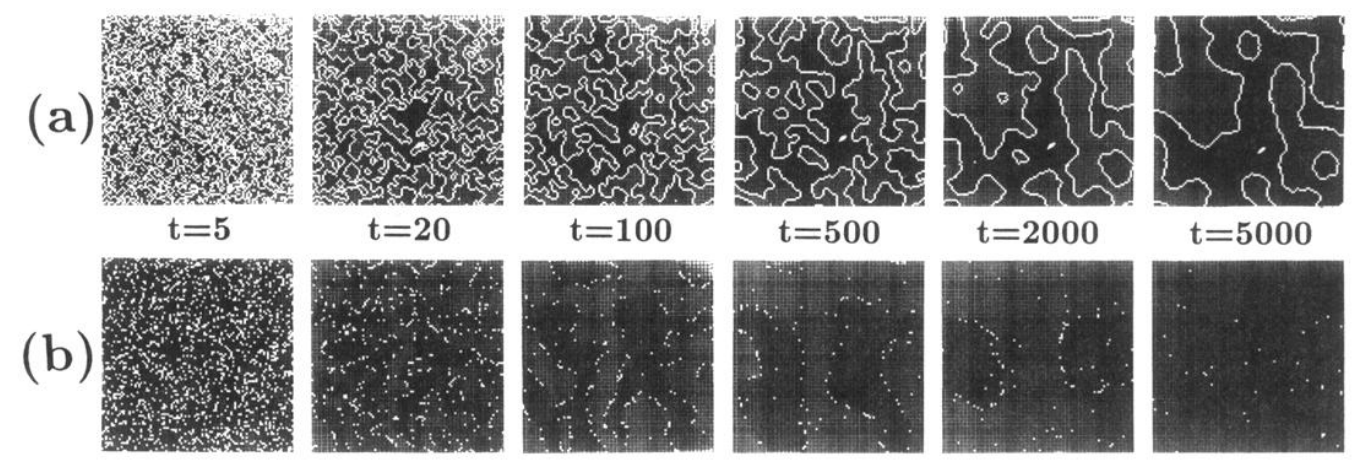

FIG. 5. Snapshots of typical microconfigurations illustrating the temporal evolution of the ordering subsequent to a deep thermal quench from $\mathrm{k}_{B} T / J=\infty$. (a) corresponds to the model with the hard condition quenched to $\mathrm{k}_{B} T / J=0.5$ and (b) to the model with the soft condition quenched to $\mathrm{k}_{B} T / J=0.1$. For both models $D=0$ in Eq. (2). The data refer to simulations on a lattice with $100 \times 100$ sites. Sites with $\sigma=0$ are indicated by blanks and sites with $\sigma= \pm 1$ are indicated in two grey tones. The time, $t$, is given in units of MCS/s. 